\title{
Examination of biomarker expressions in sepsis-related DIC patients
}

This article was published in the following Dove Press journal:

International Journal of General Medicine

\section{Michiomi Shimizu \\ Akiko Konishi \\ Shosaku Nomura}

First Department of Internal Medicine, Kansai Medical University, Hirakata, Japan
Correspondence: Shosaku Nomura First Department of Internal Medicine, Kansai Medical University, 2-3-I Shinmachi, Hirakata, Osaka 573-1191, Japan

Tel +8I 728042754

Fax +8I 72804204 I

Email shosaku-n@mbp.ocn.ne.jp
Background: Disseminated intravascular coagulation (DIC) is the main cause of death among patients with sepsis. In particular, low platelet count is predictive of poor outcome. However, the significance of platelet activation in patients with sepsis-related DIC is poorly understood. To determine the characteristics of platelet-related abnormality in patients with sepsis-related DIC, we assessed the expression levels of several biomarkers.

Methods: Plasma levels of biomarkers, including cytokines, chemokines, soluble selectins, platelet-derived microparticles (PDMPs), soluble vascular adhesion molecule 1, and high mobility group box protein 1 were measured by enzyme-linked immunosorbent assay at baseline and after 4, 7, 14, and 21 days of DIC treatment.

Results: Differences in platelet activation and in the elevation of activated platelet-related PDMPs and of soluble P-selectin were seen between patients suffering from sepsis and hematologic malignancy with DIC. In addition, the elevation of interleukin (IL)-6 and thrombopoietin (TPO) was significant in sepsis patients with DIC. Furthermore, IL-6 and TPO promoted platelet activation in vitro. Conclusion: Assessment of PDMPs, sP-selectin, IL-6, and TPO may be beneficial in the primary prevention of multi-organ failure in sepsis patients with DIC.

Keywords: disseminated intravascular coagulation, platelet activation, PDMP, sP-selectin, IL-6, TPO

\section{Introduction}

Disseminated intravascular coagulation (DIC) is frequently complicated by sepsis or hematologic malignancy, and shows fulminant clinical signs of hemorrhage. ${ }^{1}$ In addition to deterioration attributable to the original disease, DIC development doubles a specific patient's risk of death. ${ }^{1,2}$ Coagulation abnormalities and thrombocytopenia are common in DIC, and the extent of hemostatic disorders appears to correlate with disease severity. ${ }^{3}$ In particular, low platelet count is predictive of poor outcome. ${ }^{4}$ However, the significance of platelet activation in patients with sepsis-related DIC is poorly understood.

The pathophysiology of DIC is complex and involves numerous interactions between the coagulation cascade, the host immune and inflammatory system, and the damaged vascular endothelium. ${ }^{5}$ Although the diagnosis of sepsis-associated DIC is a complex clinical problem, this exaggerated response can lead to multi-organ failure, shock, and death. ${ }^{5,6}$ The severity of organ dysfunction has prognostic value, and in clinical practice is usually classified according to the Sequential Organ Failure Assessment (SOFA) score. ${ }^{7}$ Several scoring systems for DIC have been proposed and tested, with the system proposed by the International Society of Thrombosis and Hemostasis being the most widely accepted. ${ }^{8,9}$ 
In the present study, we assessed the levels of various biomarkers in patients with sepsis-related DIC, including cytokines, chemokines, soluble selectins, platelet-derived microparticles (PDMPs),${ }^{10-13}$ soluble vascular adhesion molecule 1 (sVCAM-1) protein, and high mobility group box 1 (HMGB1) protein. The purpose of the study was to clarify the characteristics of platelet-related abnormality in patients with sepsis-related DIC.

\section{Patients and methods}

\section{Patients}

The study cohort included 25 sepsis patients, 18 with and 7 without DIC, selected from those admitted to our hospital between April 2013 and August 2016. An additional group comprised 15 patients with hematological malignancy (HM; acute leukemia, malignant lymphoma, or multiple myeloma) and DIC. Three patients received angiotensin II receptor blocker in addition to statin. However, all patients not received any supplements that may effect on coagulation and inflammation, such as omega 3, vitamin E, coenzyme Q10, and vitamin K. A control group comprised 12 healthy volunteers. The study protocol was approved by our institutional review board of Kansai Medical University, and written informed consent was obtained from each patient. The 3 groups of patients and the healthy volunteers were compared in terms of age, sex, infection focus, vital signs, the Japanese Association for Acute Medicine (JAAM) DIC score ${ }^{14}$ along with the positive rate, the SOFA score ${ }^{7,15}$ on ICU admission, and therapeutic agents. Moreover, sepsis biomarkers and coagulation/fibrinolysis markers were compared between the groups.

\section{Data collection}

Baseline data for the coagulation markers, including fibrin/ fibrinogen degradation products (FDP), D-dimers, prothrombin time ratios, platelet counts, and antithrombin (AT) activity were measured before treatment. The Acute Physiology and Chronic Health Evaluation II (APACHE II) and SOFA scores were also calculated. The diagnosis of DIC was established using the JAAM and Japanese Ministry of Health and Welfare (JMHW) DIC criteria. ${ }^{14,16}$ Serial data for each coagulation marker, APACHE II score, SOFA score, JAAM-DIC, and JMHW-DIC criteria were also measured after the start of treatment.

\section{Measurement of PDMPs}

An ELISA kit used for PDMP measurements was obtained from JIMRO Co. Ltd. (Tokyo, Japan). ${ }^{17}$ The kit used 2 mono- clonal antibodies against glycoproteins CD42b and CD42a. One U/mL of PDMPs for this ELISA kit was defined as the amount of PDMPs obtained from solubilized 24,000 platelets/ $\mathrm{mL}$. Blood samples were collected from peripheral veins into vacutainers containing EDTA-anticoagulant citrate dextrose (NIPRO Co. Ltd., Osaka, Japan) using 21-gauge needles to minimize platelet activation. The samples were gently mixed by turning the tubes up-side down once or twice and then kept at room temperature for the maximum period of 2-3 hours. Immediately after centrifugation at $8,000 \times g$ for 5 minutes, $200 \mu \mathrm{L}$ was collected from the upper layer supernatant of the $2 \mathrm{~mL}$ samples to avoid contamination by platelets. The collected samples were stored at $-40^{\circ} \mathrm{C}$ until analysis. The PDMP levels were measured twice and the mean values were recorded. Furthermore, some basic studies were carried out prior to this measurement using clinical specimens.

\section{Measurement of cytokines and soluble molecules}

Blood samples from patients and controls under fasting conditions were collected into tubes with or without sodium citrate and allowed to clot at room temperature for a minimum of 1 hour. Citrated plasma or serum, respectively, was isolated by centrifugation at $1,000 \times g$ for 20 minutes at $4^{\circ} \mathrm{C}$ and stored at $-30^{\circ} \mathrm{C}$ until analyzed. Plasma concentrations of interleukin (IL)-6, thrombopoietin (TPO), soluble P-selectin (sP-selectin), sE-selectin, sL-selectin, sVCAM-1, and soluble CD40 ligand (sCD40L) were measured using monoclonal antibody-based ELISA kits (Invitrogen Inc., Camarillo, CA, USA). sVCAM-1 was identified in plasma samples. A monoclonal antibody specific for human VCAM-1 has been pre-coated onto a microplate. Samples were frozen if not analyzed shortly after collection. Multiple freeze-thaw cycles of frozen samples were unavoidable in this study. Forty assays were evaluated and the minimum detectable dose of human VCAM-1 ranged from 0.17 to $1.26 \mathrm{ng} / \mathrm{mL}$. Samples from apparently healthy volunteers were evaluated for the presence of human VCAM-1 in this assay. The range of healthy plasma was $341-897 \mathrm{ng} / \mathrm{mL}$ and SD was 132.5 ng/mL. HMGB1 was measured using an HMGB1 ELISA Kit II (Shino-test Corp., Kanagawa, Japan). The recombinant products and standard solutions provided with each kit were used as positive controls in each assay and all procedures were performed according to the manufacturers' instructions.

\section{Cutoff value of the marker}

Receiver operating characteristic analysis was performed and the optimal cutoff value of 28-day mortality was calculated 
using the marker that was selected in multivariate logistic regression.

\section{Study of platelet activation using agonists and cytokines}

To study the effect of cytokines on platelet activation and the activation-related release of PDMPs, platelet activation tests were performed using agonists, adenosine diphosphate (ADP), and collagen (Funakoshi Inc., Tokyo, Japan). IL-6 and TPO were highly purified and were checked carefully for contaminants, such as endotoxin by the supplier. Plateletrich plasma $(400 \mu \mathrm{L})$ was incubated with cytokines (IL-6, $10 \mathrm{ng} / \mathrm{mL}$, or TPO, $10 \mathrm{ng} / \mathrm{mL}$; BioSourse International Inc., Camarillo, CA, USA) and then exposed to $2 \mu \mathrm{M}$ ADP plus $1 \mu \mathrm{g} / \mathrm{mL}$ collagen. 4-(2-Hydroxyethyl)-1-piperazineethanesulfonic acid-Tyrode's buffer containing $2 \mathrm{mM}$ EDTA was added, and platelet was removed by centrifugation at $1,000 \times g$ for 15 minutes. PDMP and SCD40L levels were determined by the aforementioned ELISA methods.

\section{Treatment}

When patients met the JAAM-DIC criteria and had an AT activity level of $<70 \%$, AT concentrate (Nihon Pharmaceutical Co. Ltd., Tokyo, Japan) was administered for up to 3 consecutive days unless the patient died or treatment was stopped for any other justifiable reason..$^{18}$ The concomitant use of other anticoagulants was not prohibited, and recombinant thrombomodulin (rTM; Asahi Kasei Parma Corporation, Tokyo, Japan) was administered intravenously according to the drug manufacturer's recommendation $(0.06 \mathrm{mg} / \mathrm{kg} /$ day for 6 days by either intravenous bolus injection or intravenous infusion over 15 minutes via a catheter). ${ }^{19}$ Standard sepsis care was performed, and platelet concentrate and fresh-frozen plasma were used for substitution therapy, if necessary. ${ }^{18}$

\section{Statistics}

Data are expressed as mean \pm SD. Between-group comparisons were analyzed using the Newman-Keuls and Scheffe's tests. Correlations were assessed with Spearman's rank correlation test. The significance of differences among variables was determined by analysis of variance. $P$-values $<0.05$ were considered statistically significant. All analyses were performed using the StatFlex program (version 6).

\section{Results}

Patient demographic and clinical characteristics are shown in Table 1. Age and sex were similar in the patients (both sepsis and HM) and the healthy controls. The APACHE II scores, DIC scores, FDP, and D-dimer levels were significantly higher in patients suffering from sepsis with DIC vs sepsis without DIC. However, antithrombin III (ATIII) was significantly lower in patients with sepsis and DIC compared with sepsis without DIC.

The levels of PDMP, IL-6, TPO, HMGB1, sVCAM-1, sP-selectin, sE-selectin, and sL-selectin were compared in plasma from patients with sepsis and DIC vs healthy volunteers (Table 2). In addition, the levels of PDMP, IL-6, TPO, and $\mathrm{sP}$-selectin were found to be significantly elevated in patients with sepsis and DIC compared with HM and DIC (PDMP, $P<0.05$; IL-6, $P<0.01$; TPO, $P<0.01$; sP-selectin,

Table I Demographic and clinical characteristics of the patients and controls

\begin{tabular}{|c|c|c|c|c|}
\hline \multirow[t]{2}{*}{ Group } & \multicolumn{3}{|l|}{ Patients } & \multirow{2}{*}{$\frac{\text { Control }}{\text { Healthy persons }}$} \\
\hline & Sepsis with DIC & Sepsis without DIC & HM with DIC & \\
\hline$n$ & 18 & 7 & 15 & 12 \\
\hline Age/years & 67.5 & 63.6 & 70.1 & 65 \\
\hline Sex, male/female & $7 / 11$ & $3 / 4$ & $\mathrm{II} / 4$ & $7 / 5$ \\
\hline APACHE II score & $21.5 * *$ & 13.6 & - & - \\
\hline SOFA score & $10.8 *$ & 7.4 & - & - \\
\hline DIC score & 6.0 (JAAM)** & 2.0 (JAAM) & $6.0(\mathrm{MHH})$ & - \\
\hline PT-INR & $\mathrm{I} .4 \mathrm{I} \pm 0.1 \mathrm{I}$ & $1.31 \pm 0.09$ & $1.35 \pm 0.08$ & $\mathrm{I} .04 \pm 0.0 \mathrm{I}$ \\
\hline ATIII (\%) & $57.5 \pm 4.7^{*}$ & $68.2 \pm 5.3$ & $71.3 \pm 4.6$ & $82.5 \pm 3.1$ \\
\hline FDP $(\mu \mathrm{g} / \mathrm{mL})$ & $41.2 \pm 9.2 *$ & $26.2 \pm 5.1$ & $23.4 \pm 4.9$ & $7.2 \pm I .1$ \\
\hline D-dim $(\mu \mathrm{g} / \mathrm{mL})$ & $13.9 \pm 2.2 * *$ & $4.4 \pm 0.6$ & $11.9 \pm 2.3$ & $1.3 \pm 0.2$ \\
\hline Fbg $(\mathrm{mg} / \mathrm{mL})$ & $262 \pm 24$ & $305 \pm 31$ & $241 \pm 19$ & $286 \pm 15$ \\
\hline $\mathrm{Plt}\left(\times 10^{4} / \mu \mathrm{L}\right)$ & $7.3 \pm 2.1$ & 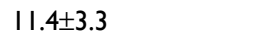 & $3.9 \pm 1.6$ & $15.2 \pm 3.5$ \\
\hline
\end{tabular}

Notes: Between-group comparisons were analyzed using the Newman-Keuls and Scheffe's tests. Data are shown as mean \pm SD. $* P<0.05 ; * * P<0.0$ I.

Abbreviations: ATIII, antithrombin III; APACHE II, Acute Physiology and Chronic Health Evaluation II; D-dim, D-dimer; DIC, disseminated intravascular coagulation; Fbg, fibrinogen; FDP, fibrin/fibrinogen degradation products; HM, hematological malignancy; JAAM, Japanese Association for Acute Medicine; JMHW, Japanese Ministry of Health and Welfare; Plt, platelet; PT-INR, prothrombin time-international normalized ratio; SOFA, Sequential Organ Failure Assessment. 
Table 2 Comparison of PDMPs, cytokines, and soluble factors between the patients and controls

\begin{tabular}{|c|c|c|c|c|}
\hline \multirow[t]{2}{*}{ Group } & \multicolumn{3}{|l|}{ Patients } & \multirow{2}{*}{$\begin{array}{l}\text { Controls } \\
\text { Healthy persons }\end{array}$} \\
\hline & Sepsis with DIC & Sepsis without DIC & HM with DIC & \\
\hline $\mathrm{n}$ & 18 & 7 & 15 & 12 \\
\hline PDMP (U/mL) & $26.8 \pm 10.2^{* 1}$ & $19.4 \pm 7.5$ & $15.3 \pm 7.4$ & $9.5 \pm 2.1$ \\
\hline IL-6 (pg/mL) & $559 \pm 284^{* 2}$ & $312 \pm 105^{* 1}$ & $105 \pm 24$ & $6.8 \pm 1.2$ \\
\hline TPO (pg/mL) & $1,866 \pm 237^{* 2}$ & $1,244 \pm 107^{* 1}$ & $425 \pm 41$ & $366 \pm 39$ \\
\hline HMGBI (ng/mL) & $35.2 \pm 19.8$ & $23.6 \pm 11.4$ & $35.9 \pm 20.2$ & $7.3 \pm 2.5$ \\
\hline sVCAM-I (pg/mL) & $1,632 \pm 508$ & $1,586 \pm 420$ & $I, 950 \pm 4 I I$ & $788 \pm 196$ \\
\hline sP-selectin (pg/mL) & $285 \pm 75^{* 1}$ & $277 \pm 96^{* 1}$ & $214 \pm 42$ & $183 \pm 26$ \\
\hline $\mathrm{sE}$-selectin $(\mathrm{pg} / \mathrm{mL})$ & $|4| \pm 76$ & $|32 \pm 4|$ & $145 \pm 39$ & $56 \pm 12$ \\
\hline sL-selectin (pg/mL) & $2,015 \pm 622$ & $1,865 \pm 513$ & $1,980 \pm 496$ & $720 \pm 217$ \\
\hline
\end{tabular}

Notes: Between-group comparisons were analyzed using the Newman-Keuls and Scheffe's tests. Data are shown as mean \pm SD.

Abbreviations: DIC, disseminated intravascular coagulation; IL-6, interleukin-6; HM, hematological malignancy; HMGBI, high-mobility group box I; PDMP, plateletderived microparticle; sE-selectin, soluble E-selectin; sL-selectin, soluble L-selectin; sP-selectin, soluble P-selectin; sVCAM-I, soluble vascular cell adhesion molecule-I; TPO, thrombopoietin.

$P<0.05)$. However, there were no significant differences in HMGB1, sVCAM-1, sE-selectin, or sL-selectin between these 2 groups.

Correlations between the 2 platelet activation markers (PDMP and sP-selectin) and cytokines are summarized in Table 3. There were significant positive correlations of platelet activation markers with IL-6 and TPO in patients with sepsis and DIC, but not HM and DIC.

We divided DIC patients into 2 subgroups according to their fundamental disease, either sepsis or HM. After DIC treatment, the sepsis subgroup showed significant reductions in plasma concentrations of HMGB1, IL-6, sE-selectin, sL-selectin, and sVCAM-1 relative to baseline $(P<0.05$ or $P<0.01$; Figures $1 \mathrm{~B}, \mathrm{C}$ and $2 \mathrm{~B}-\mathrm{D})$. However, there was no significant change from baseline in PDMP, TPO, or sP-selectin levels in these patients after treatment for DIC (Figures 1A, $\mathrm{D}$ and $2 \mathrm{~A})$.

To study the effect of cytokines on platelet activation in sepsis patients, a platelet activation test was conducted using the agonists, ADP and collagen (Figure 3). Both PDMP and sCD40L were significantly elevated in platelets stimulated by the agonists compared with unstimulated platelets (Figure $3 \mathrm{~A}$ and B). Furthermore, the addition of IL- 6 or TPO enhanced the elevation of PDMPs and SCD40L. The addition of both cytokines together significantly enhanced the elevation of PDMP, and sCD40L $(P<0.05)$ (Figure 3A and B).

\section{Discussion}

DIC complicated by leukemia is characterized by exaggerated fibrinolysis, but the consumption of ATIII is relatively low. On the other hand, DIC complicated by infection or sepsis is coagulation-predominant, resulting in the overconsumption of ATIII. The present study confirmed these
Table 3 Correlations of PDMPs and sP-selectin with cytokines in sepsis and HM patients with DIC

\begin{tabular}{llllll}
\hline Cytokines & \multicolumn{2}{l}{ PDMP } & & \multicolumn{2}{c}{ sP-selectin } \\
\cline { 2 - 3 } & $\boldsymbol{R}$ & P-value & & $\boldsymbol{R}$ & P-value \\
\hline Sepsis with DIC & & & & \\
IL-6 (pg/mL) & 0.501 & 0.010 & & 0.433 & 0.037 \\
TPO (pg/mL) & 0.454 & 0.012 & & 0.474 & 0.009 \\
HM with DIC & & & & \\
IL-6 (pg/mL) & 0.245 & 0.291 & & 0.247 & 0.234 \\
TPO (pg/mL) & 0.316 & 0.108 & 0.217 & 0.312 \\
\hline
\end{tabular}

Note: Correlations were assessed with Spearman's rank correlation test. Abbreviations: DIC, disseminated intravascular coagulation; HM, hematological malignancy; IL-6, interleukin-6; PDMP, platelet-derived microparticle; sP-selectin, soluble P-selectin; TPO, thrombopoietin.

tendencies; ATIII levels in sepsis with DIC vs HM with DIC were $57.5 \% \pm 4.7 \%$ vs $71.3 \pm 4.6 \%$. Coagulopathies are present in many sepsis patients, ${ }^{20,21}$ but the severity of sepsis-associated coagulopathy is variable, ranging up from subclinical abnormalities detectable only by a mild decrease in platelet count. ${ }^{22}$ Therefore, a low platelet count is a wellknown biomarker for sepsis disease severity.

Platelets also play a role in the pathogenesis of multiorgan failure. ${ }^{6}$ Platelet interaction with immune and endothelial cells is a well-known and conserved response against infection and sepsis. ${ }^{6}$ In particular, the elevation of neutrophils is remarkable in these diseases. Thus, platelets are also involved in the formation of neutrophil extracellular traps (NETs), which trap microorganisms and facilitate their clearance. ${ }^{23}$ The formation of NETs by platelets can promote thrombosis and contribute to organ failure. ${ }^{24}$

Platelets show complex interactions with neutrophils and the endothelium, resulting in altered glycocalyx and cytokine release. ${ }^{25}$ In these interactions, there is no doubt that $\mathrm{P}$-selectin is the most important molecule. P-selectin 
A

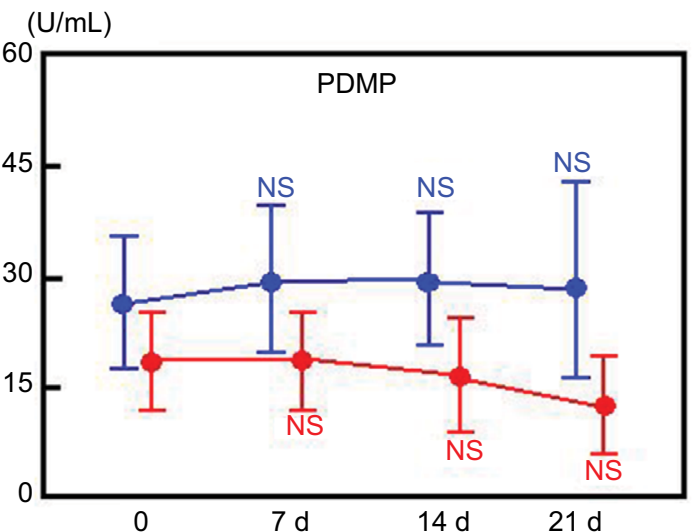

C

$(\mathrm{pg} / \mathrm{mL})$

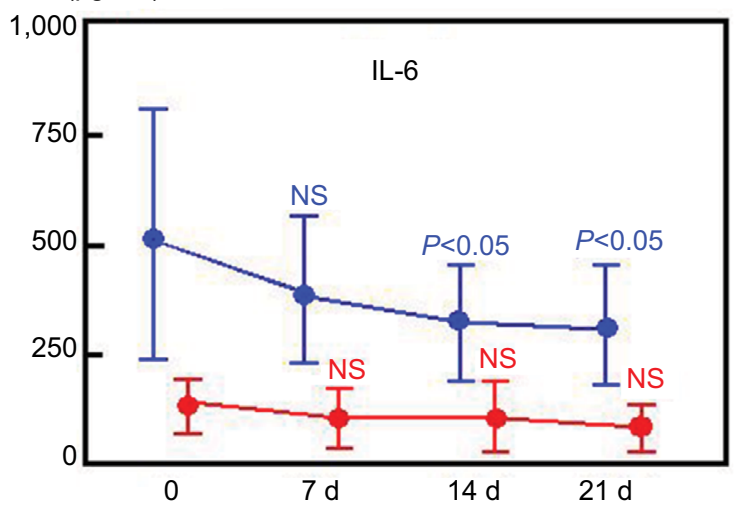

B

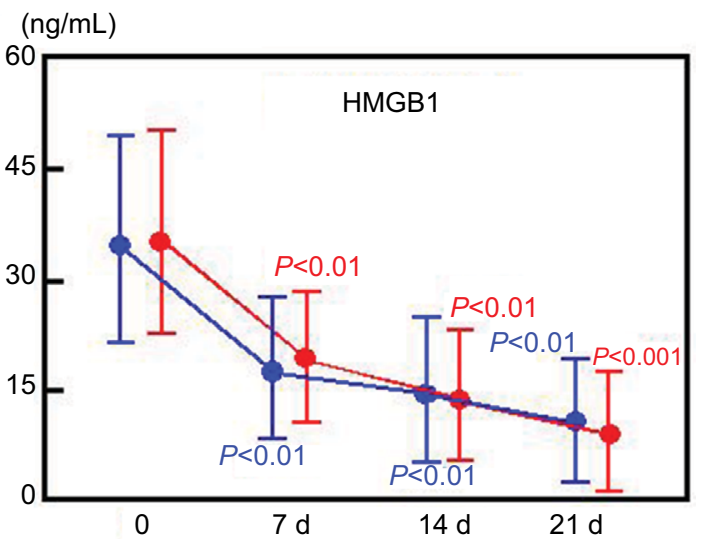

D

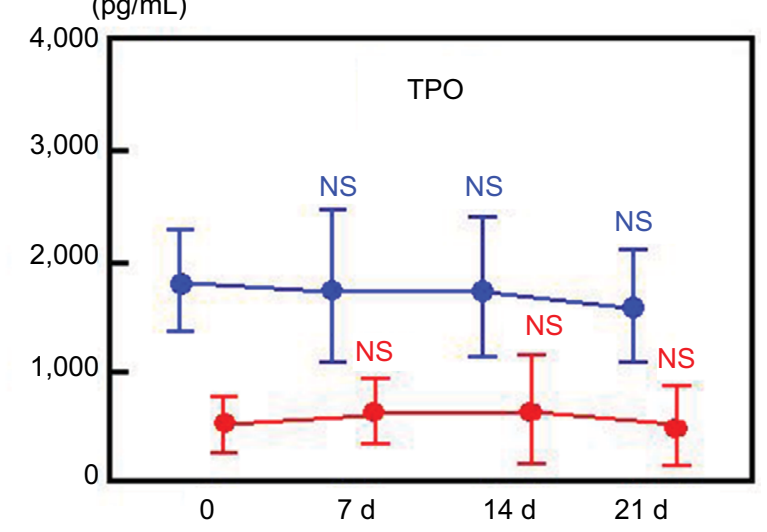

Figure I Plasma concentrations of PDMPs, HMGBI, IL-6, and TPO before and after ATIII or rTM treatment of DIC patients.

Notes: Between-group comparisons were analyzed using the Newman-Keuls and Scheffe's tests. Bars show the mean \pm SD. 0: before; d: day (after), $P$-values are for comparison with each baseline parameter ( 0 vs 7 days, 14 days, and 21 days).

Abbreviations: ATIII, antithrombin III; DIC, disseminated intravascular coagulation; HM, hematologic malignancy; HMGBI, high mobility group box I; IL-6, interleukin-6; NS, not significant; PDMP, platelet-derived microparticle; rTM, recombinant thrombomodulin; TPO, thrombopoietin.

is contained in platelet $\alpha$-granules and expressed on the membrane surface after activation. ${ }^{26}$ Greco et $\mathrm{al}^{6}$ previously reported that $\mathrm{P}$-selectin-mediated adhesion is an important platelet-endothelial cell-leukocyte interaction in sepsis. In the present study, levels of sP-selectin were significantly elevated in sepsis patients, both with and without DIC, compared with patients suffering from HM with DIC. These results are consistent with previous reports. We suspect that the low platelet count in HM patients with DIC is caused by bone marrow dysfunction. Therefore, it may be difficult to detect elevated $\mathrm{sP}$-selectin in these patients.

Another interesting result of the present study involves PDMPs. PDMPs were elevated in patients with both sepsis and $\mathrm{HM}$, compared with healthy volunteers. However, PDMPs in sepsis patients with DIC were elevated to the greatest extent. PDMPs are small vesicles released from platelet surface, which function as storage repositories for coagulation factors and cytokines. ${ }^{11,12,27}$ Elevated PDMP levels correlate with the severity of sepsis in clinical studies. ${ }^{28-30}$ Furthermore, studies investigating the effect of intravenous PDMPs in rats found that they resulted in deranged clotting, acute respiratory distress syndrome (ARDS), and a hemodynamic syndrome typical of sepsis. ${ }^{31,32}$ Our results and previous reports suggest that the elevation of PDMPs in sepsis patients with DIC originates platelet activation during the progression of sepsis.

Inflammatory cytokines such as IL-6 can modulate platelet activation. ${ }^{33}$ Interestingly, TPO has this ability as well. ${ }^{34-36}$ TPO may also be involved in platelet-leukocyte interaction and the development of organ damage in sepsis. ${ }^{6}$ TPO levels are increased in inflammatory states, ${ }^{37}$ enhancing the response of mature platelets to several agonists, increasing plateletleukocyte adhesion via $\mathrm{P}$-selectin, increasing reactive oxygen 

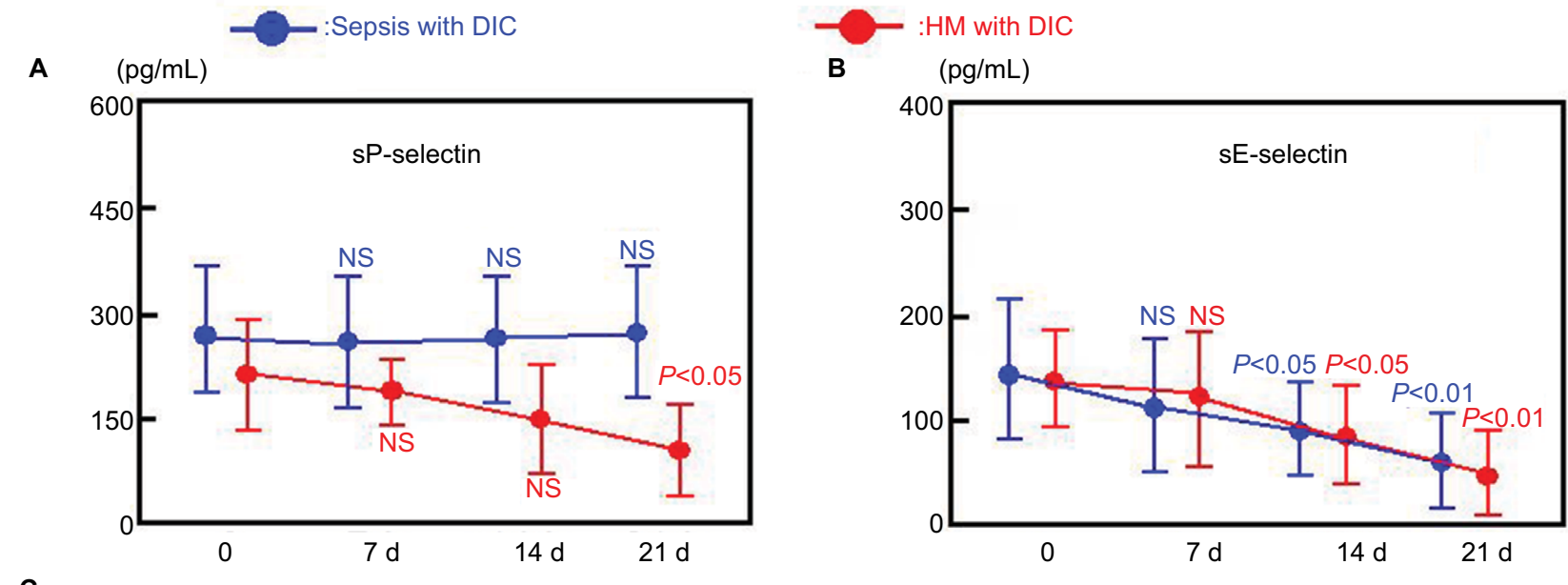

C

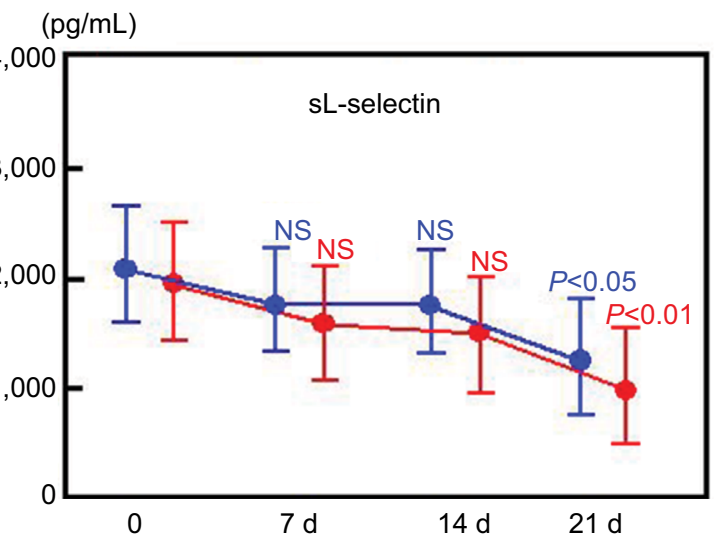

D

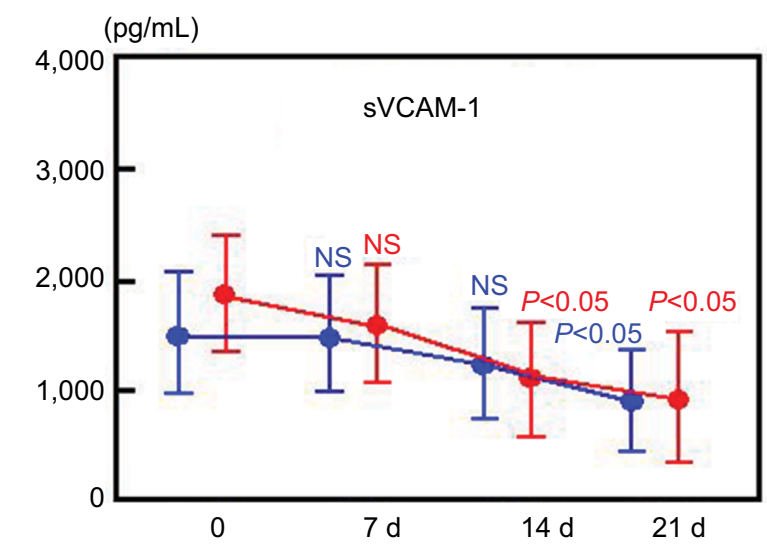

Figure 2 Plasma concentrations of sP-selectin, sE-selectin, sL-selectin, and sVCAM-I before and after ATIII or rTM treatment of DIC patients.

Notes: Between-group comparisons were analyzed using the Newman-Keuls and Scheffe's tests. Bars show the mean \pm SD. 0: before; d: day (after), P-values are for comparison with each baseline parameter ( 0 vs 7 days, 14 days, and 21 days).

Abbreviations: ATIII, antithrombin III; DIC, disseminated intravascular coagulation; HM, hematologic malignancy; NS, not significant; rTM, recombinant thrombomodulin; sP-selectin, soluble P-selectin; sE-selectin, soluble E-selectin; sL-selectin, soluble L-selectin; sVCAM-I, soluble vascular cell adhesion molecule-I.
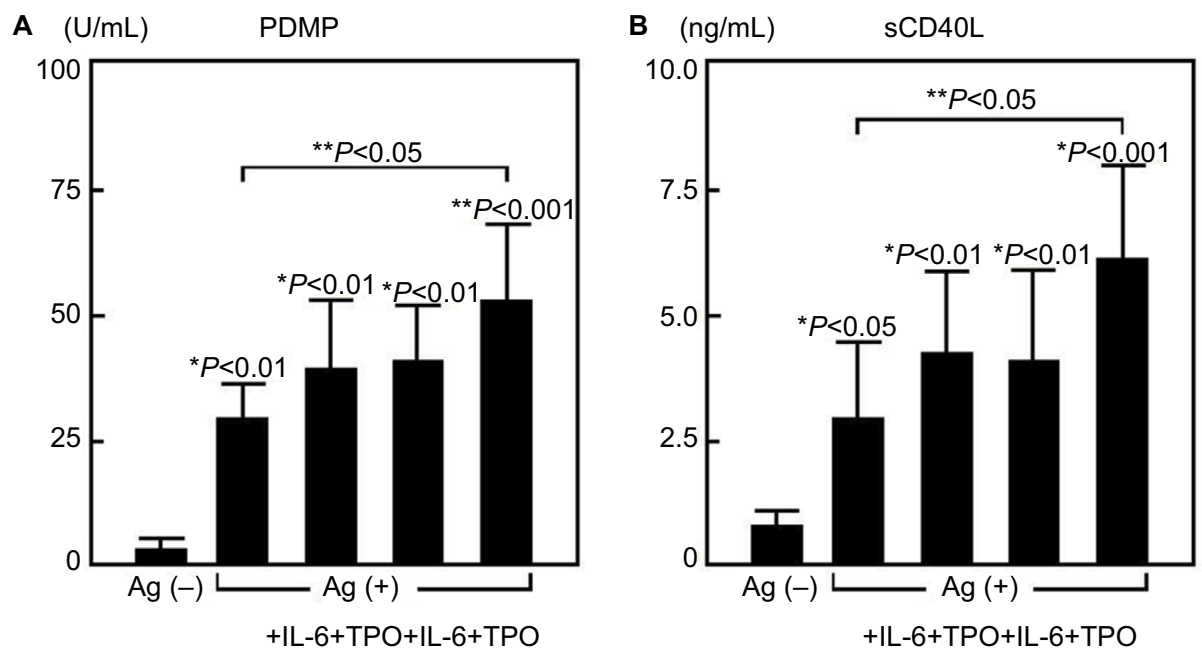

Figure 3 Study of platelet activation by agonists and cytokines.

Notes: Between-group comparisons were analyzed using the Newman-Keuls and Scheffe's tests. Bars show the mean \pm SD. These biomarkers were equally distributed. *P-values are for comparison with each baseline parameter $(\mathrm{Ag}(-)$ vs $\mathrm{Ag}(+))$. **P-values are for comparison between 2 parameters $(\mathrm{Ag}(+)$ and cytokine $(-)$ vs $\mathrm{Ag}(+)$ and IL-6 or TPO).

Abbreviations: Ag, agonist; PDMP, platelet-derived microparticle; sCD40L, soluble CD40 ligand; IL-6, interleukin-6; TPO, thrombopoietin. 
species release, and inducing IL-8 production by neutrophils and monocytes. ${ }^{38-40}$ Furthermore, significantly elevated levels of TPO have been shown in both murine and human sepsis. ${ }^{41-43}$ In the present study, IL-6 and TPO levels were both significantly elevated in patients suffering from sepsis with DIC compared with HM with DIC. We postulated that platelet activation in sepsis patients would be consequence of their elevated IL- 6 and TPO. Thus, platelet activation tests performed to measure the effect of IL- 6 and TPO on platelet activation in these patients. The addition of IL-6 and TPO significantly enhanced the elevation of PDMP and SCD40L. These results suggest that IL- 6 and TPO enhanced the platelet-endothelial cell-leukocyte interaction in sepsis, resulting in the elevation of sP-selectin, sE-selectin, and sL-selectin.

The significance of platelet activation in sepsis is also previously reported. ${ }^{6,44} 48$ ARDS is one of the most severe complications of sepsis and finally causes endothelial damage and intravascular coagulation. ${ }^{44} \mathrm{Katz}$ et $\mathrm{a}{ }^{45}$ reported that post mortem biopsies of patients who died with ARDS showed excess numbers of platelets and neutrophil deposition in pulmonary vessels. In addition, enhanced platelet activation has also been demonstrated in bronchoalveolar lavage of patients with ARDS. ${ }^{46}$ Acute kidney injury (AKI) is also a frequent complication of sepsis. ${ }^{47}$ Tókés-Füzesi et $\mathrm{al}^{48}$ reported that the correlation between PDMPs and blood urea nitrogen in AKI was indicated in sepsis patients. Therefore, these previous reports may propose as possible targets for sepsis prevention and treatment. ${ }^{49,50}$

This study has 2 potential strengths. First, we clarified differences in platelet activation and the elevation of PDMPs and of sP-selectin between patients suffering from sepsis with DIC and HM with DIC. Second, we showed that elevation of IL- 6 and TPO promotes the induction of PDMPs and sPselectin in sepsis patient with DIC. However, the study also had several limitations. First, patient numbers were small. Second, changes in clinical parameters of fibrinolysis, such as soluble-fibrin or plasminogen activator inhibitor-1 were not routinely recorded. Third, we could not identify the causes of different platelet counts in the sepsis and HM groups. HM patients had poorer values for platelet counts, suggesting that bone marrow inhibition was associated with these differences. Fourth, we could not clarify the significance of HMGB1, sP-selectin, and PDMP levels after DIC treatment. Extension of these findings in larger and more specific studies would be useful. Finally, we were unable to evaluate the therapeutic effects of ATIII and rTM using DIC scores. We suggest that the assessment of DIC scores in ATIII or rTMtreated DIC patients would be beneficial.

\section{Conclusion}

Differences were found between patients suffering from sepsis with DIC and HM with DIC in platelet activation and in the elevation of activated platelet-related PDMPs and sPselectin. In addition, IL-6 and TPO were significantly elevated in sepsis patients with DIC. IL-6 and TPO promoted platelet activation in vitro. Assessment of these biomarkers may be beneficial in the primary prevention of multi-organ failure in sepsis patients with DIC. However, larger clinical trials are required to test this hypothesis.

\section{Acknowledgments}

This study was partly supported by a grant from the Japan Foundation of Neuropsychiatry and Hematology Research, a Research Grant for Advanced Medical Care from the Ministry of Health and Welfare of Japan, and a Grant (13670760 to S.N.) from the Ministry of Education, Science and Culture of Japan. We thank Nicholas Rufaut, Phd, from Edanz Group (www.edanzediting.com/ac) for editing a draft of this manuscript.

\section{Disclosure}

The authors report no conflicts of interest in this work.

\section{References}

1. Levi M, Ten Cate H. Disseminated Intravascular Coagulation. N Engl $J$ Med Overseas Ed. 1999;341(8):586-592.

2. Saracco P, Vitale P, Scolfaro C, Pollio B, Pagliarino M, Timeus F. The coagulopathy in sepsis: significance and implications for treatment. Pediatr Rep. 2011;3(4):e30.

3. Toh CH, Dennis M. Disseminated intravascular coagulation: old disease, new hope. BMJ. 2003;327(7421):974-977.

4. Yaguchi A, Lobo FL, Vincent JL, Pradier O. Platelet function in sepsis. J Thromb Haemost. 2004;2(12):2096-2102.

5. Walborn A, Hoppensteadt D, Syed D, Mosier M, Fareed J. Biomarker Profile of Sepsis-Associated Coagulopathy Using Biochip Assay for Inflammatory Cytokines. Clin Appl Thromb Hemost. 2018;24(4): 625-632.

6. Greco E, Lupia E, Bosco O, Vizio B, Montrucchio G. Platelets and Multi-Organ Failure in Sepsis. Int J Mol Sci. 2017;18(10):E2200.

7. Howell MD, Davis AM. Management of Sepsis and Septic Shock. JAMA. 2017;317(8):847-848.

8. Taylor FB, Toh CH, Hoots WK, Wada H, Levi M, Scientific Subcommittee on Disseminated Intravascular Coagulation (DIC) of the International Society on Thrombosis and Haemostasis (ISTH). Towards definition, clinical and laboratory criteria, and a scoring system for disseminated intravascular coagulation. Thromb Haemost. 2001;86(5):1327-1330.

9. Wada H, Thachil J, di Nisio M, et al. Guidance for diagnosis and treatment of DIC from harmonization of the recommendations from three guidelines. J Thromb Haemost. 2013;11:761-767.

10. Nomura S, Ozaki Y, Ikeda Y. Function and role of microparticles in various clinical settings. Thromb Res. 2008;123(1):8-23.

11. Nomura S, Shimizu M. Clinical significance of procoagulant microparticles. J Intens Care. 2015;7:2.

12. Nomura S. Microparticle and Atherothrombotic Diseases. J Atheroscler Thromb. 2016;23(1):1-9. 
13. Miyazaki Y, Nomura S, Miyake T, et al. High shear stress can initiate both platelet aggregation and shedding of procoagulant containing microparticles. Blood. 1996;88(9):3456-3464.

14. Gando S, Iba T, Eguchi Y, et al. A multicenter, prospective validation of disseminated intravascular coagulation diagnostic criteria for critically ill patients: comparing current coagulation diagnostic criteria for critically ill patients: comparing current criteria. Crit Care Med. 2006;34:625-631.

15. Vincent JL, de Mendonça A, Cantraine F, et al. Use of the SOFA score to assess the incidence of organ dysfunction/failure in intensive care units: results of a multicenter, prospective study. Working in intensive care units: results of a multicenter, prospective study. Working group on "sepsis-related problems" of the European Society of Intensive Care Medicine. Crit Care Med. 1998;26(11):1793-1800.

16. Kobayashi N, Maekawa T, Takada M, Tanaka H, Gonmori H. Criteria for diagnosis of DIC based on the analysis of clinical and laboratory findings in 345 DIC patients collected by the Research Committee on DIC in Japan. Bibl Haematol. 1983;49(49):265-275.

17. Osumi K, Ozeki Y, Saito S, et al. Development and assessment of enzyme immunoassay for platelet-derived microparticles. Thromb Haemost. 2001;85(2):326-330.

18. Wada H, Asakura H, Okamoto K, et al. Expert consensus for the treatment of disseminated intravascular coagulation in Japan. Thromb Res. 2010;125(1):6-11.

19. Ikezoe T. Pathogenesis of disseminated intravascular coagulation in patients with acute promyelocytic leukemia, and its treatment using recombinant human soluble thrombomodulin. Int J Hematol. 2014;100(1):27-37.

20. Kinasewitz GT, Yan SB, Basson B, et al. Universal changes in biomarkers of coagulation and inflammation occur in patients with severe sepsis, regardless of causative micro-organism [ISRCTN74215569]. Crit Care. 2004;8(2):R82-R90.

21. Zeerleder S, Hack CE, Wuillemin WA. Disseminated Intravascular Coagulation in Sepsis. Chest. 2005;128(4):2864-2875.

22. Koyama K, Madoiwa S, Nunomiya S, et al. Combination of thrombinantithrombin complex, plasminogen activator inhibitor-1, and protein $\mathrm{C}$ activity for early identification of severe coagulopathy in initial phase of sepsis: a prospective observational study. Crit Care. 2014;18(1):R13.

23. Clark SR, Ma AC, Tavener SA, et al. Platelet TLR4 activates neutrophil extracellular traps to ensnare bacteria in septic blood. Nat Med. 2007;13(4):463-469.

24. Andrews RK, Arthur JF, Gardiner EE. Neutrophil extracellular traps (NETs) and the role of platelets in infection. Thromb Haemost. 2014;112(4):659-665.

25. Ince C, Mayeux PR, Nguyen T, et al. THE ENDOTHELIUM IN SEPSIS Shock. 2016;45(3):259-270.

26. Yeo EL, Sheppard JA, Feuerstein IA. Role of P-selectin and leukocyte activation in polymorphonuclear cell adhesion to surface adherent activated platelets under physiologic shear conditions (an injury vessel wall model. Blood. 1994;83(9):2498-2507.

27. Burnier L, Fontana P, Kwak BR, Angelillo-Scherrer A. Cell-derived microparticles in haemostasis and vascular medicine. Thromb Haemost. 2009;101(3):439-451.

28. Tőkés-Füzesi M, Woth G, Ernyey B, et al. Microparticles and acute renal dysfunction in septic patients. J Crit Care. 2013;28(2):141-147.

29. Woth G, Tökés-Füzesi M, Magyarlaki T, Kovács GL, Vermes I, Mühl D. Activated platelet-derived microparticle numbers are elevated in patients with severe fungal (Candida albicans) sepsis. Ann Clin Biochem. 2012;49(Pt 6):554-560.

30. Ohuchi M, Fujino K, Kishimoto T, et al. Association of the Plasma Platelet-Derived Microparticles to Platelet Count Ratio with Hospital Mortality and Disseminated Intravascular Coagulopathy in Critically Ill Patients. J Atheroscler Thromb. 2015;22(8):773-782.
31. Li H, Meng X, Liang X, Gao Y, Cai S. Administration of microparticles from blood of the lipopolysaccharide-treated rats serves to induce pathologic changes of acute respiratory distress syndrome. Exp Biol Med. 2015;240(12):1735-1741.

32. Mortaza S, Martinez MC, Baron-Menguy C, et al. Detrimental hemodynamic and inflammatory effects of microparticles originating from septic rats. Crit Care Med. 2009;37(6):2045-2050.

33. Oleksowicz L, Mrowiec Z, Isaacs R, Dutcher JP, Puszkin E. Morphologic and ultrastructural evidence of interleukin-6 induced platelet activation. Am J Hematol. 1995;48(2):92-99.

34. Oda A, Miyakawa Y, Druker BJ, et al. Thrombopoietin primes human platelet aggregation induced by shear stress and by multiple agonists. Blood. 1996;87(11):4664-4670.

35. Nomura S, Nakamura T, Cone J, Tandon NN, Kambayashi J. Cytometric analysis of high shear-induced platelet microparticles and effect of cytokines on microparticle generation. Cytometry. 2000;40(3): 173-181.

36. Nomura S, Inami N, Kanazawa S, Iwasaka T, Fukuhara S. Elevation of platelet activation markers and chemokines during peripheral blood stem cell harvest with G-CSF. Stem Cells. 2004;22(5):696-703.

37. Lupia E, Goffi A, Bosco O, Montrucchio G. Thrombopoietin as biomarker and mediator of cardiovascular damage in critical diseases. Mediators Inflamm. 2012;2012:390892.

38. Montrucchio G, Brizzi MF, Calosso G, Marengo S, Pegoraro L, Camussi G. Effects of recombinant human megakaryocyte growth and development factor on platelet activation. Blood. 1996;87(7):2762-2768.

39. Tibbles HE, Navara CS, Hupke MA, Vassilev AO, Uckun FM. Thrombopoietin induces p-selectin expression on platelets and subsequent platelet/leukocyte interactions. Biochem Biophys Res Commun. 2002;292(4):987-991.

40. Lupia E, Bosco O, Bergerone S, et al. Thrombopoietin contributes to enhanced platelet activation in patients with unstable angina. $\mathrm{J} \mathrm{Am} \mathrm{Coll}$ Cardiol. 2006;48(11):2195-2203.

41. Zakynthinos SG, Papanikolaou S, Theodoridis T, et al. Sepsis severity is the major determinant of circulating thrombopoietin levels in septic patients. Crit Care Med. 2004;32(4):1004-1010.

42. Lupia E, Bosco O, Mariano F, et al. Elevated thrombopoietin in plasma of burned patients without and with sepsis enhances platelet activation. J Thromb Haemost. 2009;7(6):1000-1008.

43. Segre E, Pigozzi L, Lison D, et al. May thrombopoietin be a useful marker of sepsis severity assessment in patients with SIRS entering the emergency department? Clin Chem Lab Med. 2014;52(10):1479-1483.

44. Yadav H, Kor DJ. Platelets in the pathogenesis of acute respiratory distress syndrome. Am J Physiol Lung Cell Mol Physiol. 2015;309(9): L915-L923.

45. Katz JN, Kolappa KP, Becker RC. Beyond thrombosis: the versatile platelet in critical illness. Chest. 2011;139(3):658-668.

46. Carvalho AC, Demarinis S, Scott CF, Silver LD, Schmaier AH, Colman RW. Activation of the contact system of plasma proteolysis in the adult respiratory distress syndrome. J Lab Clin Med. 1988;112(2):270-277.

47. Singbartl K, Ley K. Leukocyte recruitment and acute renal failure. $J$ Mol Med. 2004;82(2):91-101.

48. Tökés-Füzesi M, Woth G, Ernyey B, et al. Microparticles and acute renal dysfunction in septic patients. J Crit Care. 2013;28(2): 141-147.

49. Valerio-Rojas JC, Jaffer IJ, Kor DJ, Gajic O, Cartin-Ceba R. Outcomes of severe sepsis and septic shock patients on chronic antiplatelet treatment: a historical cohort study. Crit Care Res Pract. 2013;2013:782573.

50. Wang L, Li H, Gu X, Wang Z, Liu S, Chen L. Effect of Antiplatelet Therapy on Acute Respiratory Distress Syndrome and Mortality in Critically Ill Patients: A Meta-Analysis. PLoS One;11(5):e0154754. 
The International Journal of General Medicine is an international, peer-reviewed open-access journal that focuses on general and internal medicine, pathogenesis, epidemiology, diagnosis, monitoring and treatment protocols. The journal is characterized by the rapid reporting of reviews, original research and clinical studies across all disease areas.
The manuscript management system is completely online and includes a very quick and fair peer-review system, which is all easy to use. Visit http://www.dovepress.com/testimonials.php to read real quotes from published authors. 